MMCA-97 Conference, pp. 130-137

R. Čiegis (Ed)

(C) 1997 Vilniaus Gedimino technikos universitetas

\title{
DECOUPLING OF IMPULSIVE DIFFERENTIAL EQUATIONS
}

\author{
ANDREJS REINFELDS ${ }^{1}$ \\ Institute of Mathematics, \\ Latvian Academy of Sciences and University of Latvia, \\ Akadèmijas laukums 1, LV-1524 Rīga, LATVIA; \\ e-mail: reinf@com.latnet.lv
}

\section{INTRODUCTION}

Impulsive differential equations provide an adequate mathematical model of evolutionary processes that suddenly change their state at certain moments. V. Lakshmikantham, D. D. Bainov and P. S. Simeonov [5] as well as A. M. Samoilenko and N. A. Perestyuk [12] had published monographs dedicated to this subject.

The equivalence problem in the theory of ordinary differential equations were explored by D. M. Grobman [2], P. Hartman [3] and other mathematicians $[4,15]$. A. Reinfelds [6-11] and L. Sermone [6-7,13-14] and D. D. Bainov, S. I. Kostadinov and Nguyen Van Minh [1] began to discuss the equivalence problem of impulsive differential equations. In the present paper a reduction theorem for systems of impulsive differential equations in a Banach space is proven assuming that the system splits into two parts and has invariant manifold.

\section{STATEMENT OF THE THEOREMS}

Let $\mathbf{U}$ be a Banach space. Consider two systems of impulsive differential equations

$$
d u / d t=P(t, u),\left.\Delta u\right|_{t=\tau_{i}}=S_{i}\left(u\left(\tau_{i}-0\right)\right)
$$

and

$$
d u / d t=Q(t, u),\left.\Delta u\right|_{t=\tau_{i}}=T_{i}\left(u\left(\tau_{i}-0\right)\right)
$$

that satisfy the conditions of the existence and uniquenesses theorem. We assume that maximum interval of the existence of the solutions is $\mathbf{R}$. Let

\footnotetext{
${ }^{1}$ This work was partly supported by Latvian Council of Science under Grant 96. 0692.
} 
$\phi\left(\cdot, t_{0}, u_{0}\right): \mathbf{R} \rightarrow \mathbf{U}$ and $\psi\left(\cdot, t_{0}, u_{0}\right): \mathbf{R} \rightarrow \mathbf{U}$ be the solutions of the above systems, respectively. Suppose that there is a function $e: \mathbf{U} \rightarrow \mathbf{R}$ such that

$$
\max \left\{|P(t, u)-Q(t, u)|, \sup _{i}\left|S_{i}(u)-T_{i}(u)\right|\right\} \leq e(u) .
$$

Definition. Two systems of impulsive differential equations (1) and (2) are dynamically equivalent in the large if there exists a map $H: \mathbf{R} \times \mathbf{U} \rightarrow \mathbf{U}$ and a positive constant $c$ such that:

(i) $H(t, \cdot): \mathbf{U} \rightarrow \mathbf{U}$ is a homeomorphism;

(ii) $H\left(t, \phi\left(t, t_{0}, u_{0}\right)\right)=\psi\left(t, t_{0}, H\left(t_{0}, u_{0}\right)\right)$ for all $t \in \mathbf{R}$;

(iii) $\max \left\{|H(t, u)-u|,\left|H^{-1}(t, u)-u\right|\right\} \leq c e(u)$;

We remark that in the case of classical global Grobman-Hartman theorem $[2,3]$ for autonomous differential equations the corresponding function $e(x)=$ $a>0$ and appropriate constant $c$ depend on linear truncation only.

Let $\mathbf{U}=\mathbf{X} \times \mathbf{Y}, \mathcal{L}(\mathbf{X})$ and $\mathcal{L}(\mathbf{Y})$ be the Banach spaces of linear bounded operators. Consider the following system of impulsive differential equations

$$
\begin{cases}d x / d t & =A(t) x+f(t, x, y) \\ d y / d t & =B(t) y+g(t, x, y) \\ \left.\Delta x\right|_{t=\tau_{i}} & =x\left(\tau_{i}+0\right)-x\left(\tau_{i}-0\right) \\ & =C_{i} x\left(\tau_{i}-0\right)+p_{i}\left(x\left(\tau_{i}-0\right), y\left(\tau_{i}-0\right)\right) \\ \left.\Delta y\right|_{t=\tau_{i}} & =y\left(\tau_{i}+0\right)-y\left(\tau_{i}-0\right) \\ & =D_{i} y\left(\tau_{i}-0\right)+q_{i}\left(x\left(\tau_{i}-0\right), y\left(\tau_{i}-0\right)\right)\end{cases}
$$

where:

(i) the maps $A: \mathbf{R} \rightarrow \mathcal{L}(\mathbf{X})$ and $B: \mathbf{R} \rightarrow \mathcal{L}(\mathbf{Y})$ are locally integrable in the Bochner sense;

(ii) the maps $f: \mathbf{R} \times \mathbf{X} \times \mathbf{Y} \rightarrow \mathbf{X}$ and $g: \mathbf{R} \times \mathbf{X} \times \mathbf{Y} \rightarrow \mathbf{Y}$ are locally integrable in the Bochner sense with respect to $t$ for fixed $x$ and $y$, and, in addition, they satisfy the estimates

$$
\begin{gathered}
\left|f(t, x, y)-f\left(t, x^{\prime}, y^{\prime}\right)\right| \leq \varepsilon\left(\left|x-x^{\prime}\right|+\left|y-y^{\prime}\right|\right), \\
\left|g(t, x, y)-g\left(t, x^{\prime}, y^{\prime}\right)\right| \leq \varepsilon\left(\left|x-x^{\prime}\right|+\left|y-y^{\prime}\right|\right), \\
\sup _{t, y}|f(t, 0, y)|<+\infty
\end{gathered}
$$

(iii) $i \in \mathbf{Z}, C_{i} \in \mathcal{L}(\mathbf{X}), D_{i} \in \mathcal{L}(\mathbf{Y})$, the maps $p_{i}: \mathbf{X} \times \mathbf{Y} \rightarrow \mathbf{X}, q_{i}: \mathbf{X} \times \mathbf{Y} \rightarrow \mathbf{Y}$ satisfy the estimates

$$
\begin{gathered}
\left|p_{i}(x, y)-p_{i}\left(x^{\prime}, y^{\prime}\right)\right| \leq \varepsilon\left(\left|x-x^{\prime}\right|+\left|y-y^{\prime}\right|\right) \\
\left|q_{i}(x, y)-q_{i}\left(x^{\prime}, y^{\prime}\right)\right| \leq \varepsilon\left(\left|x-x^{\prime}\right|+\left|y-y^{\prime}\right|\right) \\
\quad \sup _{i, y}\left|p_{i}(0, y)\right|<+\infty
\end{gathered}
$$


(iv) the maps $(x, y) \mapsto\left(x+C_{i} x+p_{i}(x, y), y+D_{i} y+q_{i}(x, y)\right), x \mapsto x+C_{i} x$ are homeomorphisms;

(v) the moments $\tau_{i}$ of impulse effect form a strictly increasing sequence

$$
\ldots<\tau_{-2}<\tau_{-1}<\tau_{0}<\tau_{1}<\tau_{2}<\ldots
$$

where the limit points may be only $\pm \infty$.

Let $U(t, \tau)$ and $V(t, \tau)$ be the Cauchy evolutionary operators of the linear impulsive systems

$$
\begin{cases}d x / d t & =A(t) x \\ \left.\Delta x\right|_{t=\tau_{i}} & =C_{i} x\left(\tau_{i}-0\right)\end{cases}
$$

and

$$
\begin{cases}d y / d t & =B(t) y \\ \left.\Delta y\right|_{t=\tau_{i}} & =D_{i} y\left(\tau_{i}-0\right)\end{cases}
$$

respectively. Let the operators satisfy the estimates

$$
\begin{gathered}
\nu=\max \left\{\sup _{t} \int_{-\infty}^{t}|V(t, \tau)||U(\tau, t)| d \tau+\sup _{t} \sum_{\tau_{i} \leq t}\left|V\left(t, \tau_{i}\right)\right|\left|U\left(\tau_{i}-0, t\right)\right|,\right. \\
\left.\sup _{t} \int_{t}^{+\infty}|V(\tau, t)||U(t, \tau)| d \tau+\sup _{t} \sum_{t<\tau_{i}}\left|V\left(\tau_{i}-0, t\right)\right|\left|U\left(t, \tau_{i}\right)\right|\right\}<+\infty, \\
\mu=\sup _{t}\left\{\int_{t}^{+\infty}|U(t, \tau)| d \tau+\sum_{t<\tau_{i}}\left|U\left(t, \tau_{i}\right)\right|\right\}<+\infty .
\end{gathered}
$$

Let $\Phi\left(\cdot, t_{0}, x_{0}, y_{0}\right)=\left(x\left(\cdot, t_{0}, x_{0}, y_{0}\right), y\left(\cdot, t_{0}, x_{0}, y_{0}\right)\right): \mathbf{R} \rightarrow \mathbf{X} \times \mathbf{Y}$ be the solution of system $(3)$, where $\Phi\left(t_{0}+0, t_{0}, x_{0}, y_{0}\right)=\left(x_{0}, y_{0}\right)$. At the break points $\tau_{i}$ the values for all solutions are taken at $\tau_{i}+0$ unless otherwise specified. For short, we will use the notation $\Phi(t)=(x(t), y(t))$.

ThEOREM 1. Let $4 \varepsilon \nu \leq 1$ and $2 \varepsilon \mu<1+\sqrt{1-4 \varepsilon \nu}$. Then there exists a piecewise continuous map $h: \mathbf{R} \times \mathbf{Y} \rightarrow \mathbf{X}$ with the following properties:

(i) $h\left(t, y\left(t, t_{0}, h\left(t_{0}, y_{0}\right), y_{0}\right)\right)=x\left(t, t_{0}, h\left(t_{0}, y_{0}\right), y_{0}\right)$ for all $t \in \mathbf{R}$;

(ii) $\left|h\left(t, y_{0}\right)-h\left(t, y_{0}^{\prime}\right)\right| \leq \lambda\left|y_{0}-y_{0}^{\prime}\right|$;

(iii) $\int_{-\infty}^{t_{0}}\left|V\left(t_{0}, t\right)\right|\left|x\left(t, t_{0}, x_{0}, y_{0}\right)-h\left(t, y\left(t, t_{0}, x_{0}, y_{0}\right)\right)\right| d t$

$+\sum_{\tau_{i}<t}^{-\infty}\left|V\left(t_{0}, \tau_{i}\right)\right|\left|x\left(\tau_{i}-0, t_{0}, x_{0}, y_{0}\right)-h\left(\tau_{i}-0, y\left(\tau_{i}-0, t_{0}, x_{0}, y_{0}\right)\right)\right|$

$\leq \nu(1-\varepsilon(1+\lambda) \nu)^{-1}\left|x_{0}-h\left(t_{0}, y_{0}\right)\right|$, 
where $\lambda=(2 \varepsilon \nu)^{-1}(1-2 \varepsilon \nu-\sqrt{1-4 \varepsilon \nu})$.

Suppose there exists maps $f_{0}: \mathbf{R} \times \mathbf{X} \rightarrow \mathbf{X}$ and $p_{i 0}: \mathbf{X} \rightarrow \mathbf{X}$ locally integrable in the Bochner sense with respect to $t$ for fixed $x$ and, in addition, they satisfy the estimates:

$$
\begin{gathered}
\left|f_{0}(t, x)-f_{0}\left(t, x^{\prime}\right)\right| \leq \varepsilon\left|x-x^{\prime}\right|, \\
\left|p_{i 0}(x)-p_{i 0}\left(x^{\prime}\right)\right| \leq \varepsilon\left|x-x^{\prime}\right|, \\
\sup _{t, x, y}\left|f(t, x, y)-f_{0}(t, x)\right|<+\infty \\
\sup _{i, x, y}\left|p_{i}(x, y)-p_{i 0}(x)\right|<+\infty
\end{gathered}
$$

and besides the maps $x \mapsto x+C_{i} x+p_{i 0}(x)$ are homeomorphisms. Next, consider a reduced system of impulsive differential equations

$$
\left\{\begin{array}{l}
d x / d t=A(t) x+f_{0}(t, x) \\
d y / d t=B(t) y+g(t, h(t, y), y) \\
\left.\Delta x\right|_{t=\tau_{i}}=C_{i} x\left(\tau_{i}-0\right)+p_{i 0}\left(x\left(\tau_{i}-0\right)\right) \\
\left.\Delta y\right|_{t=\tau_{i}}=D_{i} y\left(\tau_{i}-0\right)+q_{i}\left(h\left(\tau_{i}-0, y\left(\tau_{i}-0\right)\right), y\left(\tau_{i}-0\right)\right)
\end{array}\right.
$$

Let $\Psi\left(\cdot, t_{0}, x_{0}, y_{0}\right)=\left(x_{0}\left(\cdot, t_{0}, x_{0}\right), y_{0}\left(\cdot, t_{0}, y_{0}\right)\right): \mathbf{R} \rightarrow \mathbf{X} \times \mathbf{Y}$ be a solution of system (4), where $\Psi\left(t_{0}+0, t_{0}, x_{0}, y_{0}\right)=\left(x_{0}, y_{0}\right)$. For short, we will use the notation $\Psi(t)=\left(x_{0}(t), y_{0}(t)\right)$.

TheOREM 2. Let $4 \varepsilon \nu<1$ and $2 \varepsilon \mu<1+\sqrt{1-4 \varepsilon \nu}$. Then systems (3) and (4) are dynamically equivalent in the large.

The system (4) splits into two parts. The first part of them does not contain the variable $y$, while the second part is independent of $x$. This result allows one to replace the given system by a much simpler one.

\section{PROOF OF THEOREM 2}

Step by step we shall prove the theorem. Let $\mathbf{P C}(\mathbf{R} \times \mathbf{X} \times \mathbf{Y}, \mathbf{X})$ be a set of maps that are continuous for $(t, x, y) \in\left[\tau_{i}, \tau_{i+1}\right) \times \mathbf{X} \times \mathbf{Y}$ and have discontinuities of the first kind for $t=\tau_{i}$.

Step 1. The spaces

$$
\mathcal{K}=\left(\begin{array}{l|l}
k \in \mathbf{P C}(\mathbf{R} \times \mathbf{X} \times \mathbf{Y}, \mathbf{X}) & \sup _{t, x, y}|k(t, x, y)|<+\infty
\end{array}\right)
$$

and

$$
\mathcal{L}_{1}=\left(\begin{array}{l|l}
l \in \mathbf{P C}(\mathbf{R} \times \mathbf{X} \times \mathbf{Y}, \mathbf{Y}) & \left.\sup _{t, x, y} \frac{|l(t, x, y)|}{|x-h(t, y)|}<+\infty\right)
\end{array}\right.
$$


equipped with the norms

$$
\|k\|=\sup _{t, x, y}|k(t, x, y)| \text { and }\|l\|=\sup _{t, x, y} \frac{|l(t, x, y)|}{|x-h(t, y)|}
$$

are Banach spaces, respectively. There exists a unique solution $\left(k_{1}, l_{1}\right) \in$ $\mathcal{K} \times \mathcal{L}_{1}$ of the functional equations

$$
\begin{gathered}
k_{1}\left(t_{0}, x_{0}, y_{0}\right)=\int_{t_{0}}^{+\infty} U\left(t_{0}, \tau\right)\left(f(\tau, \Phi(\tau))-f_{0}\left(\tau, x(\tau)+k_{1}(\tau, \Phi(\tau))\right)\right) d \tau \\
+\sum_{t_{0}<\tau_{i}} U\left(t_{0}, \tau_{i}\right)\left(p_{i}\left(\Phi\left(\tau_{i}-0\right)\right)-p_{i 0}\left(x\left(\tau_{i}-0\right)+k_{1}\left(\tau_{i}-0, \Phi\left(\tau_{i}-0\right)\right)\right)\right), \\
l_{1}\left(t_{0}, x_{0}, y_{0}\right) \\
=\int_{-\infty}^{t_{0}} V\left(t_{0}, \tau\right)\left(g\left(\tau, h\left(\tau, y(\tau)+l_{1}(\tau, \Phi(\tau))\right), y(\tau)+l_{1}(\tau, \Phi(\tau))\right)-g(\tau, \Phi(\tau))\right) d \tau \\
+\sum_{\tau_{i} \leq t_{0}} V\left(t_{0}, \tau_{i}\right)\left(q _ { i } \left(h\left(\tau_{i}-0, y\left(\tau_{i}-0\right)+l_{1}\left(\tau_{i}-0, \Phi\left(\tau_{i}-0\right)\right)\right), y\left(\tau_{i}-0\right)\right.\right. \\
\left.\left.+l_{1}\left(\tau_{i}-0, \Phi\left(\tau_{i}-0\right)\right)\right)-q_{i}\left(\Phi\left(\tau_{i}-0\right)\right)\right) .
\end{gathered}
$$

Let $H\left(t_{0}, x_{0}, y_{0}\right)=\left(x_{0}+k_{1}\left(t_{0}, x_{0}, y_{0}\right), y_{0}+l_{1}\left(t_{0}, x_{0}, y_{0}\right)\right)$. We get for all $t \in \mathbf{R}$ that

$$
H\left(t, \Phi\left(t, t_{0}, x_{0}, y_{0}\right)\right)=\Psi\left(t, t_{0}, H\left(t_{0}, x_{0}, y_{0}\right)\right) .
$$

Step 2. The set

$$
\mathcal{L}_{1}(\lambda)=\left(l \in \mathcal{L}_{1}|| l(t, x, y)-l\left(t, x^{\prime}, y\right)|\leq \lambda| x-x^{\prime} \mid\right)
$$

is a closed subset of the Banach space $\mathcal{L}_{1}$. There exists a unique solution $\left(k_{2}, l_{2}\right) \in \mathcal{K} \times \mathcal{L}_{1}(\lambda)$ of the functional equations

$$
\begin{gathered}
k_{2}\left(t_{0}, x_{0}, y_{0}\right)=\int_{t_{0}}^{+\infty} U\left(t_{0}, \tau\right)\left(f_{0}\left(\tau, x_{0}(\tau)\right)-f\left(\tau, x_{0}(\tau)+k_{2}(\tau, \Psi(\tau)), y_{0}(\tau)\right.\right. \\
\left.\left.\quad+l_{2}\left(\tau, x_{0}(\tau)+k_{2}(\tau, \Psi(\tau)), y_{0}(\tau)\right)\right)\right) d \tau \\
+\sum_{t_{0}<\tau_{i}} U\left(t_{0}, \tau_{i}\right)\left(p_{i 0}\left(x_{0}\left(\tau_{i}-0\right)\right)-p_{i}\left(x_{0}\left(\tau_{i}-0\right)+k_{2}\left(\tau_{i}-0, \Psi\left(\tau_{i}-0\right)\right), y_{0}\left(\tau_{i}-0\right)\right.\right. \\
\left.\left.+l_{2}\left(\tau_{i}-0, x_{0}\left(\tau_{i}-0\right)+k_{2}\left(\tau_{i}-0, \Psi\left(\tau_{i}-0\right)\right), y_{0}\left(\tau_{i}-0\right)\right)\right)\right)
\end{gathered}
$$




$$
\begin{gathered}
l_{2}\left(t_{0}, x_{0}, w_{0}\right)=\int_{-\infty}^{t_{0}} V\left(t_{0}, \tau\right)\left(g\left(\tau, \eta(\tau), y_{0}(\tau)+l_{2}\left(\tau, \eta(\tau), y_{0}(\tau)\right)\right)\right. \\
\left.-g\left(\tau, h\left(\tau, y_{0}(\tau)\right), y_{0}(\tau)\right)\right) d \tau \\
+\sum_{\tau_{i} \leq t_{0}} V\left(t_{0}, \tau_{i}\right)\left(q_{i}\left(\eta\left(\tau_{i}-0\right), y_{0}\left(\tau_{i}-0\right)+l_{2}\left(\tau_{i}-0, \eta\left(\tau_{i}-0\right), y_{0}\left(\tau_{i}-0\right)\right)\right)\right. \\
\left.-q_{i}\left(h\left(\tau_{i}-0, y_{0}\left(\tau_{i}-0\right)\right), y_{0}\left(\tau_{i}-0\right)\right)\right) \\
+\sum_{t_{0}}^{t} U(t, \tau) f\left(\tau, \eta(\tau), y_{0}(\tau)+l_{2}\left(\tau, \eta(\tau), y_{0}(\tau)\right)\right) d \tau \\
+\sum_{t_{0}<\tau_{i} \leq t} U\left(t, \tau_{i}\right) p_{i}\left(\eta\left(\tau_{i}-0\right), y_{0}\left(\tau_{i}-0\right)+l_{2}\left(\tau_{i}-0, \eta\left(\tau_{i}-0\right), y_{0}\left(\tau_{i}-0\right)\right)\right)
\end{gathered}
$$

Let $G\left(t_{0}, x_{0}, y_{0}\right)=\left(x_{0}+k_{2}\left(t_{0}, x_{0}, y_{0}\right), y_{0}+l_{2}\left(t_{0}, x_{0}+k_{2}\left(t_{0}, x_{0}, y_{0}\right), y_{0}\right)\right)$. We get for all $t \in \mathbf{R}$ that

$$
G\left(t, \Psi\left(t, t_{0}, x_{0}, y_{0}\right)\right)=\Phi\left(t, t_{0}, G\left(t_{0}, x_{0}, y_{0}\right)\right)
$$

Step 3. The space

$$
\mathcal{L}_{2}=\left(\begin{array}{l|l}
l \in \mathbf{P C}(\mathbf{R} \times \mathbf{X} \times \mathbf{Y}, \mathbf{Y}) & \left.\sup _{t, x, y} \frac{|l(t, x, y)|}{\left|x+k_{2}(t, x, y)-h(t, y)\right|}<+\infty\right)
\end{array}\right)
$$

equipped with the norm

$$
\|l\|=\sup _{t, x, y} \frac{|l(t, x, y)|}{\left|x+k_{2}(t, x, y)-h(t, y)\right|}
$$

is a Banach space. There exists a unique solution $\left(k_{3}, l_{3}\right) \in \mathcal{K} \times \mathcal{L}_{2}$ of the functional equations

$$
\begin{gathered}
k_{3}\left(t_{0}, x_{0}, y_{0}\right)=\int_{t_{0}}^{+\infty} U\left(t_{0}, \tau\right)\left(f_{0}\left(\tau, x_{0}(\tau)\right)-f_{0}\left(\tau, x_{0}(\tau)+k_{3}(\tau, \Psi(\tau))\right)\right) d \tau \\
+\sum_{t_{0}<\tau_{i}} U\left(t_{0}, \tau_{i}\right)\left(p_{i 0}\left(x_{0}\left(\tau_{i}-0\right)\right)-p_{i 0}\left(x_{0}\left(\tau_{i}-0\right)+k_{3}\left(\tau_{i}-0, \Psi\left(\tau_{i}-0\right)\right)\right)\right), \\
l_{3}\left(t_{0}, x_{0}, y_{0}\right)=\int_{-\infty}^{t_{0}} V\left(t_{0}, \tau\right)\left(g \left(\tau, h\left(\tau, y_{0}(\tau)+l_{3}(\tau, \Psi(\tau))\right), y_{0}(\tau)\right.\right. \\
\left.\left.+l_{3}(\tau, \Psi(\tau))\right)-g\left(\tau, h\left(\tau, y_{0}(\tau)\right), y_{0}(\tau)\right)\right) d \tau
\end{gathered}
$$




$$
\begin{gathered}
+\sum_{\tau_{i} \leq t_{0}} V\left(t_{0}, \tau_{i}\right)\left(q _ { i } \left(h\left(\tau_{i}-0, y_{0}\left(\tau_{i}-0\right)+l_{3}\left(\tau_{i}-0, \Psi\left(\tau_{i}-0\right)\right)\right), y_{0}\left(\tau_{i}-0\right)\right.\right. \\
\left.\left.+l_{3}\left(\tau_{i}-0, \Psi\left(\tau_{i}-0\right)\right)\right)-q_{i}\left(h\left(\tau_{i}-0, y_{0}\left(\tau_{i}-0\right)\right), y_{0}\left(\tau_{i}-0\right)\right)\right) .
\end{gathered}
$$

Step 4. The identity

$$
H\left(t_{0}, G\left(t_{0}, x_{0}, y_{0}\right)\right)=\left(x_{0}, y_{0}\right)
$$

holds true.

Step 5. The space

$\mathcal{L}_{3}=\left(l \in \mathbf{P C}(\mathbf{R} \times \mathbf{X} \times \mathbf{Y} \times \mathbf{X}, \mathbf{Y}) \mid \sup _{t, x, y, w} \frac{|l(t, x, y, w)|}{\max (|x-h(t, y)|,|x-w|)}<+\infty\right)$

equipped with the norm

$$
\|l\|=\sup _{t, x, y, w} \frac{|l(t, x, y, w)|}{\max (|x-h(t, y)|,|x-w|)}
$$

is a Banach space. The set

$$
\mathcal{L}_{3}(\lambda)=\left(l \in \mathcal{L}_{3}|| l(t, x, y, w)-l\left(t, x, y, w^{\prime}\right)|\leq \lambda| w-w^{\prime} \mid\right)
$$

is a closed subset of $\mathcal{L}_{3}$. There exists a unique solution $\left(k_{4}, l_{4}\right) \in \mathcal{K} \times \mathcal{L}_{3}(\lambda)$ of the functional equations

$$
\begin{gathered}
k_{4}\left(t_{0}, x_{0}, y_{0}\right)=\int_{t_{0}}^{+\infty} U\left(t_{0}, \tau\right)(f(\tau, \Phi(\tau)) \\
\left.-f\left(\tau, x(\tau)+k_{4}(\tau, \Phi(\tau)), y(\tau)+l_{4}\left(\tau, \Phi(\tau), x(\tau)+k_{4}(\tau, \Phi(\tau))\right)\right)\right) d \tau \\
+\sum_{t_{0}<\tau_{i}} U\left(t_{0}, \tau_{i}\right)\left(p_{i}\left(\Phi\left(\tau_{i}-0\right)\right)-p_{i}\left(x\left(\tau_{i}-0\right)\right.\right. \\
\left.\left.+k_{4}\left(\tau_{i}-0, \Phi\left(\tau_{i}-0\right)\right), y\left(\tau_{i}-0\right)+l_{4}\left(\tau_{i}-0, \Phi\left(\tau_{i}-0\right), x\left(\tau_{i}-0\right)+k_{4}\left(\tau_{i}-0, \Phi\left(\tau_{i}-0\right)\right)\right)\right)\right) \\
\eta(t)=U\left(t, t_{0}\right) w_{0}+\int_{t_{0}}^{t} U(t, \tau) g\left(\tau, \eta(\tau), y(\tau)+l_{4}(\tau, \Phi(\tau), \eta(\tau))\right) d \tau \\
+\sum_{t_{0}<\tau_{i} \leq t} U\left(t, \tau_{i}\right) q_{i}\left(\eta\left(\tau_{i}-0\right), y\left(\tau_{i}-0\right)+l_{4}\left(\tau_{i}-0, \Phi\left(\tau_{i}-0\right), \eta\left(\tau_{i}-0\right)\right)\right), \\
l_{4}\left(t_{0}, x_{0}, y_{0}, w_{0}\right)=\int_{-\infty}^{t_{0}} V\left(t_{0}, \tau\right)\left(g\left(\tau, \eta(\tau), y(\tau)+l_{4}(\tau, \Phi(\tau), \eta(\tau))\right)-g(\tau, \Phi(\tau))\right) d \tau
\end{gathered}
$$


$+\sum_{\tau_{i} \leq t_{0}} V\left(t_{0}, \tau_{i}\right)\left(q_{i}\left(\eta\left(\tau_{i}-0\right), y\left(\tau_{i}-0\right)+l_{4}\left(\tau_{i}-0, \Phi\left(\tau_{i}-0\right), \eta\left(\tau_{i}-0\right)\right)\right)-q_{i}\left(\Phi\left(\tau_{i}-0\right)\right)\right)$.

Step 6. The following identity

$$
G\left(t_{0}, H\left(t_{0}, x_{0}, y_{0}\right)\right)=\left(x_{0}, y_{0}\right)
$$

holds true.

We get that $H\left(t_{0}, \cdot\right)$ is homeomorphism establishing dynamical equivalence of systems (3) and (4) in the large. It is easy to verify that if the system (3) of differential equations is autonomous and without impulse effect, then the maps $h, H$ and $G$ are independent of $t_{0} \in \mathbf{R}$. Let us note that in our case $e(x, y)=a+\varepsilon|x|$, where $a$ is some positive constant. Thus the proof of the theorem is complete.

\section{REFERENCES}

[1] D. D. Bainov, S. I. Kostadinov and Nguyen Van Minh, Dichotomies and Integral Manifolds of Impulsive Differential Equations, Science Culture Technology Publishing, Singapore, 1994.

[2] D. M. Grobman, Topological classification of neighborhoods of a singularity in $n-$ space, Mat. Sb., 56(1), 1962, P. 77 - 94. (In Russian).

[3] P. Hartman, Ordinary Differential Equations, Birkhäuser, Boston, 1982.

[4] U. Kirchgraber and K. J. Palmer, Geometry in the Neighborhood of Invariant Manifolds of Maps and Flows and Linearization, Pitman Res. Notes Math. Ser., 233 , Longman, New York, 1991.

[5] V. Lakshmikantham, D. D. Bainov, and P. S. Simeonov, Theory of Impulsive Differential Equations, World Scientific, River Edge, New Jersy, 1989.

[6] A. Reinfelds and L. Sermone, Equivalence of differential equations with impulse action, Latv. Univ. Zinātn. Raksti, 553, 1990, P. 124 - 130. (In Russian).

[7] A. Reinfelds and L. Sermone, Equivalence of nonlinear differential equations with impulse effect in Banach space, Latv. Univ. Zinātn. Raksti, 577, 1992, P. $68-73$.

[8] A. Reinfelds, Existence of central manifold for differential equations with impulses in a Banach space, Latv. Univ. Zinātn. Raksti, 577, 1992, P. $81-88$.

[9] A. Reinfelds, Reduction theorem for differential equations with impulse effect in a Banach space, J. Math. Anal. Appl., 203, 1996(1), P. $187-210$.

[10] A. Reinfelds, Decoupling of impulsive differential equations in a Banach space, In C. Costanda, J. Saranen and S. Seikkala (eds), Integral Methods in Science and Engineering, 1, 1996, Addison Wesley Longman (in print).

[11] A. Reinfelds, Dynamical equivalence of impulsive differential equations, Nonlinear Anal., (in print).

[12] A. M. Samoilenko and N. A. Perestyuk, Differential Equations with Impulse Effect, Vishcha Shkola, Kiev, 1987. (In Russian).

[13] L. Sermone, Equivalence of differential equations with impulse effect, Proc. Latv. Acad. Sci. Sect., B 2(559), 1994, P. $78-80$.

[14] L. Sermone, Reduction of differential equations with impulse effect, J. Appl. Math. Stochastic Anal., 10(1), 1997, P. $79-87$.

[15] A. N. Shoshitaishvili, Bifurcations of topological type at singular points of parametrized vectorfields, Functional Anal. Appl., 6, 1972, P. 169 - 170. 